\title{
Study of Biases in the Prescription of SSRIs with Respect to Pharmacokinetic, Applicative, Demographic, and Commercial Aspects
}

\author{
Stuti Mittal", Haresh Raulgaonkar \\ School of Pharmacy and Technology Management, Narsee Monjee Institute of Management Studies, Mumbai, India \\ Email address: \\ stutimittal96@gmail.com (S. Mittal) \\ ${ }^{*}$ Corresponding author \\ To cite this article: \\ Stuti Mittal, Haresh Raulgaonkar. Study of Biases in the Prescription of SSRIs with Respect to Pharmacokinetic, Applicative, Demographic, \\ and Commercial Aspects. International Journal of Business and Economics Research. Vol. 8, No. 3, 2019, pp. 97-107. \\ doi: $10.11648 /$ j.ijber.20190803.13
}

Received: March 3, 2019; Accepted: May 31, 2019; Published: June 12, 2019

\begin{abstract}
Objective: To study the biases in the prescription of Selective Serotonin Reuptake Inhibitors and the variation between the literature and the perception of doctors prescribing SSRIs. Methodology: The five drugs under study were sertraline, fluoxetine, fluvoxamine, paroxetine, escitalopram. The factors influencing the psychiatrist's perceptions/ practice considered in this study are: A) Pharmacokinetic factors of the drug (Contra indications, Drug efficacy, Onset of action, Duration of action and the Bioavailability) B) Uses of the drug (for Social Anxiety Disorder, Generalized Anxiety Disorder, Major Depressive Disorder, Obsessive Compulsive Disorder and Post-Traumatic Stress Disorder) C) Side effects (Somnolence, Anxiety, Tremors, Anorexia and sexual dysfunction) D) Patient's Demographics (Gender, Age group, Special GroupsGeriatrics and Adolescents) E) Commercial aspects (Retail Price, Availability, Brand Value of the Parent Brand and Interpersonal Skills of the Medical Representative). Discussion: Contra-Indications: Theoretically, all SSRIs have the same number of contra-indications, since they have the same mechanism of action, though fluoxetine and paroxetine have a relatively higher potential of drug-drug interactions. Nonetheless, escitalopram was perceived to have the lowest, while fluvoxamine was perceived to have the highest number of contra-indications. Duration of action: There is no set difference between the duration of action of the 5 drugs under study, although, according to secondary research, $1 / 3^{\text {rd }}$ of the total therapeutic effect at six weeks was apparent by the $1^{\text {st }}$ week of treatment. Also, fluoxetine has the highest half-life at $2-4$ days, while that of paroxetine is the least, at 16 hours. Yet, based on the study, Sertraline was perceived to have the highest duration of action. Demographics: Although there was no literature found to back this bias, Sertraline was the most preferred drug for us in geriatric patients, while Paroxetine was the least preferred. For adolescent populations, Paroxetine was the most preferred and Fluoxetine was the least preferred. Conclusion: There exists a gap between the theoretical evidence and the practical observations about the pharmacokinetics, uses and side effects of the 5 SSRIs under study. For example, sertraline, a drug proven to be useful in the treatment of depression, has an incorrect bias of causing anxiety as a side effect. This results in psychiatrists not prescribing an effective drug due to a bias with no theoretical basis, thus resulting in poorer treatment outcomes for the patient. For healthcare organizations, it means that they are losing out on almost half the potential patient population. Therefore, it would be useful for them to undertake communication campaigns that revolved around thwarting such biases, thus resulting in better treatment choices for patients and improved health outcomes.
\end{abstract}

Keywords: Antidepressants, Selective Serotonin Reuptake Inhibitor, Pharmacokinetics of SSRIs

\section{Introduction}

Depression is a common illness characterized by persistent sadness and a loss of interest in activities that one normally enjoys, accompanied by an inability to carry out daily activities, for at least two weeks. For the individual suffering from depression may experience loss of energy, indecisiveness, restlessness, variation in appetite, variation in 
sleep, reduction in ability to concentrate, feeling of being non worthy, feeling of hopelessness, judging oneself guilty and going to an extent of considering self-harm and even suicide. [1]

Different categories of anxiety disorders such as major depressive disorder (MDD), social anxiety disorder (SAD), generalized anxiety disorder (GAD), obsessive compulsive disorder (OCD), post-traumatic stress disorder (PTSD) have characteristic symptoms and cognitions. There exists common co-existence of depression with all of the above categories of disorders. All above disorders respond to psychological treatments and drugs such as selective serotonin reuptake inhibitors (SSRIs), benzodiazepines and other sedative like compounds. [2]

Selective serotonin reuptake inhibitors or serotonergic antidepressants are the class of drugs that are typically used as first line antidepressant treatment. Introduced more than a decade ago in the primary care setting, the exact mechanism of action for these drugs is still unknown, though the entire class is presumed to be functioning with a similar mechanism. The introduction of SSRIs into the antidepressive treatment area simplified the treatment options for depression manifold, since they have a broader therapeutic range. They also show lesser side effects, and do not have related complications such as urinary retention and slower cardiac conduction. Thus, patients undergoing SSRI treatment seldom need constant therapeutic dose management. [3]

\section{Literature Review}

\subsection{Pharmacokinetic Aspects of SSRIs}

\subsubsection{Mechanism of Action}

Though the exact mechanism of selective serotonin reuptake inhibitors is unknown, they are thought to increase the extracellular level of serotonin by reducing its reabsorption into a presynaptic cell. They thus increase the serotonin levels in the synaptic cleft that can now bind to the postsynaptic receptor. The selectivity for monoamine oxidase (MAO) transporters varies between SSRIs, with them having weak affinity for dopamine and norepinephrine transporters. [4]

\subsubsection{Onset of Action}

Conventional wisdom suggested that treatment with SSRIs needs to be continued for at least 2 to 4 weeks for visible therapeutic effects. Recent studies concluded that treatment is associated with symptomatic improvement in depression by the end of the $1^{\text {st }}$ week of use. [5]

\subsubsection{Bioavailability and Efficacy}

Figure 1 shows the oral bioavailability of SSRIs. The absorption of SSRIs is usually good, with peak plasma concentrations observed after about four to six hours of oral administration. The absolute bioavailability of escitalopram is about $100 \%$, whereas that of the other molecules is comparatively lesser due to the fact that they undergo substantial first pass metabolism. The apparent oral clearance values after single dose vary from $26 \mathrm{~L} / \mathrm{h}$ for escitalopram to $167 \mathrm{~L} / \mathrm{h}$ for paroxetine. Despite of repeated studies, no reliable evidence exists for establishing a relationship between the clinical efficacy and the plasma concentrations of any of the SSRIs. [6]

\subsubsection{Duration of Action}

Recently, an analysis of placebo-controlled trials of SSRIs showed that the therapeutic response of the drugs is highest in the first week. The benefits of the treatment gradually decline over the successive weeks of administration. $1 / 3^{\text {rd }}$ of the total therapeutic effect at six weeks was apparent by the $1^{\text {st }}$ week of treatment. Being a placebo controlled trial, it was apparent that the improvement was unlikely to be occurring due to any placebo effect. [7]

\subsection{Uses of SSRIs}

Figure 1 shows the various uses of the SSRIs. Depending on the SSRI molecule, they can be used to treat a number of disorders such as major depressive disorder (MDD), social anxiety disorder (SAD), generalized anxiety disorder (GAD), obsessive compulsive disorder (OCD), post-traumatic stress disorder (PTSD) and for the treatment of certain phobias. [8]

\subsection{Side Effects and Tolerability}

Figure 1 shows the various side effects, specific to each of the SSRIs. Gastrointestinal (GIT) disturbances are the most frequent side effect of SSRI use. During phase IV clinical trials it was established that fluvoxamine has the highest reports of GIT disturbances as a side effect, while fluoxetine and sertraline are associated with anxiety, insomnia and agitation. Based on the tolerability, we have:

Escitalopram $>$ fluoxetine $>$ sertraline $>$ paroxetine $>$ fluvoxamine

Paroxetine and fluvoxamine were also reported to have the highest amount of side effects, and thus had the highest discontinuation rates as well. The most troubling adverse effects during the course of long-term SSRI therapy were reported to be weight gain, sexual dysfunction, and sleep disturbance. [9]

\subsection{Demographic Factors}

\subsubsection{Gender}

There exists a genuine gender difference in depressive disorders having its roots in adverse childhood or adolescence experiences along with the coping strength of the individual. But whether this has any role in the prescription of SSRIs is a matter of investigation. [10]

\subsubsection{Special Groups}

Patients in the geriatric age group are usually administered many medications on a daily basis. Due to aging, their body is less efficient at elimination of these drugs. It also has increased sensitivity towards the pharmacotherapeutic side effects and the adverse effects that may occur due to drugdrug interactions. Since they co-administer many drugs at a 
time, the chances of drug-drug interactions to occur increases manifold. [11]

Children and adolescents have a delicate system, which is yet developing. Thus, chronic administration of drugs or coadministration of drugs may have extreme or unforeseen adverse reactions to the body. Thus, for both these age groups, it is essential to carefully monitor the drug combinations being administered. If required, therapeutic drug monitoring should be employed. Furthermore, in both these cases, only specialists who are well trained in geriatric, pediatric and adolescent psychiatry should be prescribing SSRIs. [12]

\subsection{Commercial Aspects of SSRIs}

\subsubsection{Current Market Scenario}

According to the Drugs and Cosmetics Act, 1940, antidepressants are classified as Schedule $\mathrm{X}$ drugs. For this reason, they cannot be advertised or marketed to the end consumers in any way, since they can be procured only through a prescription of a registered medical practitioner. Detailing about antidepressants to doctors and psychiatrists is a tough business. The detailing process cannot simply rely on characteristics such as brand value. Unless a new and improved molecule is introduced into the market by any organization, psychiatrists are less likely to switch brands having the same SSRI molecule. But sadly, the amount of resources being invested into R\&D of SSRIs is constantly declining, and thus there have not been any new SSRI molecules introduced into the market since 2002 (escitalopram). [13]

\subsubsection{Generic Competition}

Since 2001, most of the major SSRI molecules have been going off patent. Prozac (fluoxetine) was the first to lose its patent in the year 2001, followed by Paxil (paroxetine) in 2003, Celexa (citalopram) in 2004 and finally Zoloft (sertraline) in 2006. The parent companies of Paxil and Prozac came out with modified versions of the molecule in order to extend the term of their patents under the Patent Term Restoration Act, but by the year 2012, generic version of all the SSRI molecules had reached the market. The flood of generics in the market made it tougher for the branded generics to survive. Thus, it became essential for these companies to employ better detailing skills than the generic versions, to ensure that psychiatrists stayed loyal to their brands. [14]

\subsubsection{Cost of Drugs}

The cost of the drug is an important factor during prescription of antidepressant drugs. Other than fluoxetine and escitalopram, none of the other SSRI molecules have any price cap attached to them. Even in the case of fluoxetine and escitalopram, fixed dose combinations do not come under the purview of the National List Of Essential Medicines, only those formulations containing only the aforementioned APIs do. Since manufacturers are free to market their brands at any price, the cost of the drug for the consumer becomes one of the major factors in increasing the saliency of a particular brand in the market. [15]

Table 1. Comparison of escitalopram, fluoxetine, paroxetine, fluvoxamine and sertraline based on their pharmacokinetic properties, uses and side effects. [11].

\begin{tabular}{|c|c|c|c|c|c|}
\hline & Escitalopram & Fluoxetine & Paroxetine & Fluvoxamine & Sertraline \\
\hline $\begin{array}{l}\text { Drug interaction } \\
\text { potential }\end{array}$ & Relatively low & High & Moderate to high & Relatively low & Relatively low \\
\hline $\begin{array}{l}\text { Most common side } \\
\text { effects }\end{array}$ & $\begin{array}{l}\text { Nausea Insomnia } \\
\text { Diarrhoea } \\
\text { Headache }\end{array}$ & $\begin{array}{l}\text { Nausea Headache } \\
\text { Insomnia } \\
\text { Nervousness } \\
\text { Anxiety Drowsiness } \\
\text { Anorexia Diarrhoea }\end{array}$ & $\begin{array}{l}\text { Nausea Drowsiness } \\
\text { Headache Dry mouth } \\
\text { Dizziness Weakness Fatigue } \\
\text { Sexual dysfunction Increased } \\
\text { sweating }\end{array}$ & $\begin{array}{l}\text { Nausea Vomiting Weight loss } \\
\text { Agitation Nervousness } \\
\text { Anxiety Insomnia } \\
\text { Somnolence Tremors } \\
\text { Headache Dizziness }\end{array}$ & $\begin{array}{l}\text { Nausea Headache } \\
\text { Insomnia } \\
\text { Diarrhoea Dry } \\
\text { mouth Sexual } \\
\text { dysfunction }\end{array}$ \\
\hline Half-life & 27-32 hours & 2-4 days & 20 hours (highly variable) & 16 hours (variable) & 26 hours \\
\hline Oral bioavailability & $51-93 \%$ & $70 \%$ & $50 \%$ & $53 \%$ & $20-36 \%$ \\
\hline Uses & MDD GAD & MDD OCD PTSD & MDD OCD PD & OCD MDD SAD PTSD & $\begin{array}{l}\text { MDD SAD OCD } \\
\text { PTSD }\end{array}$ \\
\hline
\end{tabular}

\section{Objectives}

The objective of the study was to capture the biases of practicing psychiatrist in the city of Mumbai, India with respect to the prescription of SSRIs considering pharmacokinetic, applicative, demographic, and commercial aspects.

\section{Methodology}

To meet the objectives a primary data collection was planned to cover maximum possible practicing psychiatrist in Mumbai, India. A structured questionnaire employing 5 point Likert agreement scale was used and responses were collected by visiting the doctors in their practicing places. A list of 186 psychiatrists practising in Mumbai was obtained from the database of online app 'Practo' (https://www.practo.com) which gives the contact details of the required speciality of the doctors and is freely accessible to all for booking appointments for doctor consultation. Out of these 60 were selected randomly as target respondents to be contacted using the free random number generator available from Google. Out of these finally 49 psychiatrists agreed to meet and gave their responses in span of 3 months from $14^{\text {th }}$ July 2016 to $14^{\text {th }}$ October 2016 .

The collected data is analyzed using IBM SPSS version 19 using T test, One way ANOVA and Ranking tools.

Generalized hypothesis and decision rule for $\mathrm{T}$ test for 5 point Likert scale,

Null hypothesis, Ho: $\mu=3$ i.e. neutral preference towards 
use of the drug molecule brand under consideration in reference to the variable under consideration.

Alternate hypothesis, $\mathrm{H} 1: \mu \neq 3$ i.e. non-neutral preference towards use of the drug molecule brand under consideration in reference to the variable under consideration.

Generalized hypothesis for One way ANOVA test:

Null hypothesis, Ho: No significant difference among the 5 antidepressant SSRI drug molecule brands under study in reference to the variable under consideration.

Alternate hypothesis, H1: Significant difference among the 5 antidepressant SSRI drug molecule brands under study in reference to the variable under consideration.

Significance level used for the study: 5\%

\section{Results}

\subsection{Pharmacokinetic Properties}

Refer to figure 1. The experience / perceptions / opinions of psychiatrists on different characteristics of drugs under study were significant. Figure 2 shows the \% score given by psychiatrists to drugs under study, the clustered bar charts and one way ANOVA for the particular pharmacokinetic properties. In One way ANOVA within the group, significant mean differences were observed in only two pharmacokinetic characteristics of the drugs: 'Low contra indications' and 'Long duration of action'.

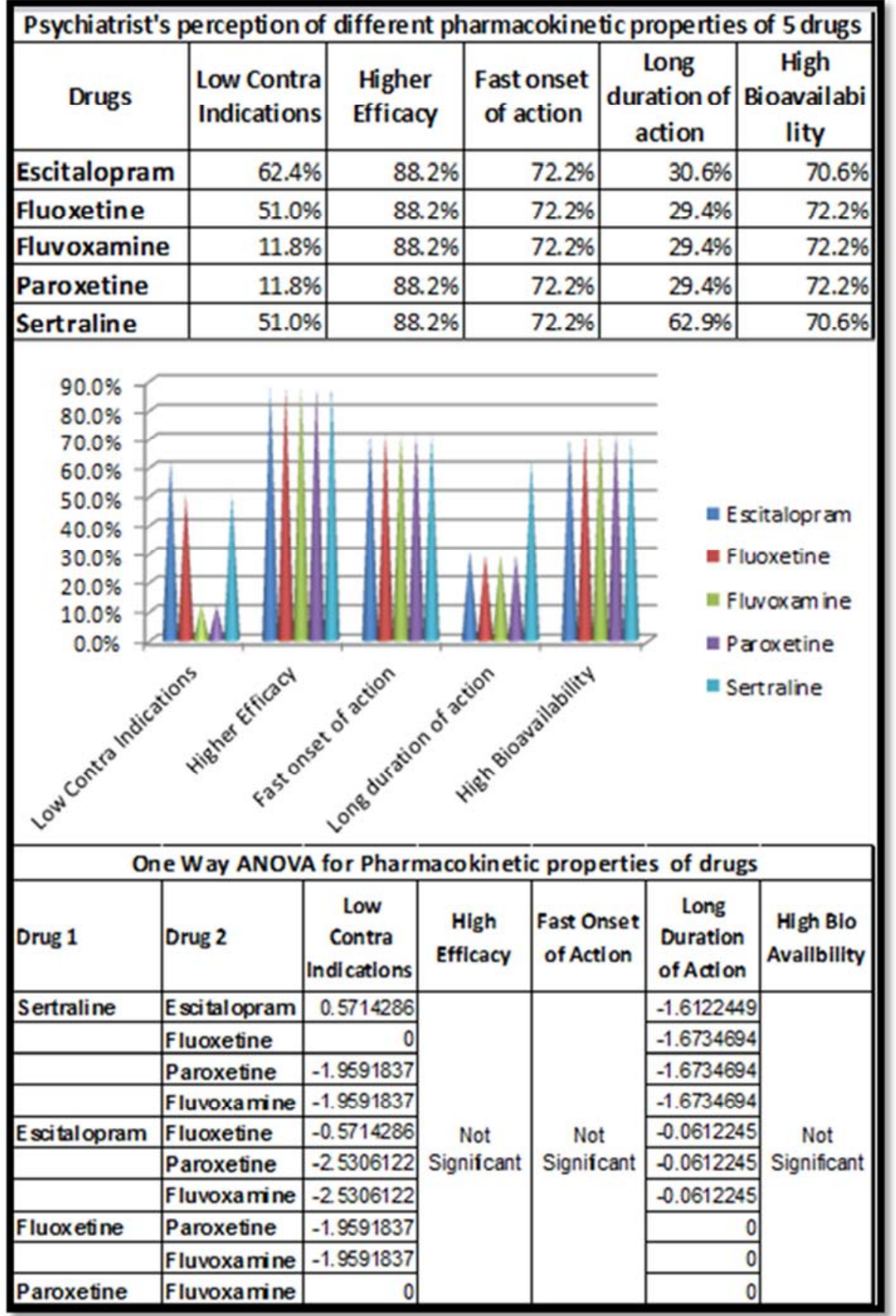

Figure 1. Scores given by Psychiatrists, clustered bar charts and one way ANOVA for different pharmacokinetic properties of drugs. 


\subsection{Uses of SSRIs}

Refer to figure 2. For each type of disorder under study, the preferences for all drugs were significant. Figure shows the \% preference score for the use of the particular drug by psychiatrists, the clustered bar charts and the one way ANOVA for the drugs preferred for the particular disorder type. Significant mean differences were observed in preferences for the 5 drugs when variances within the group were tested using One way ANOVA.

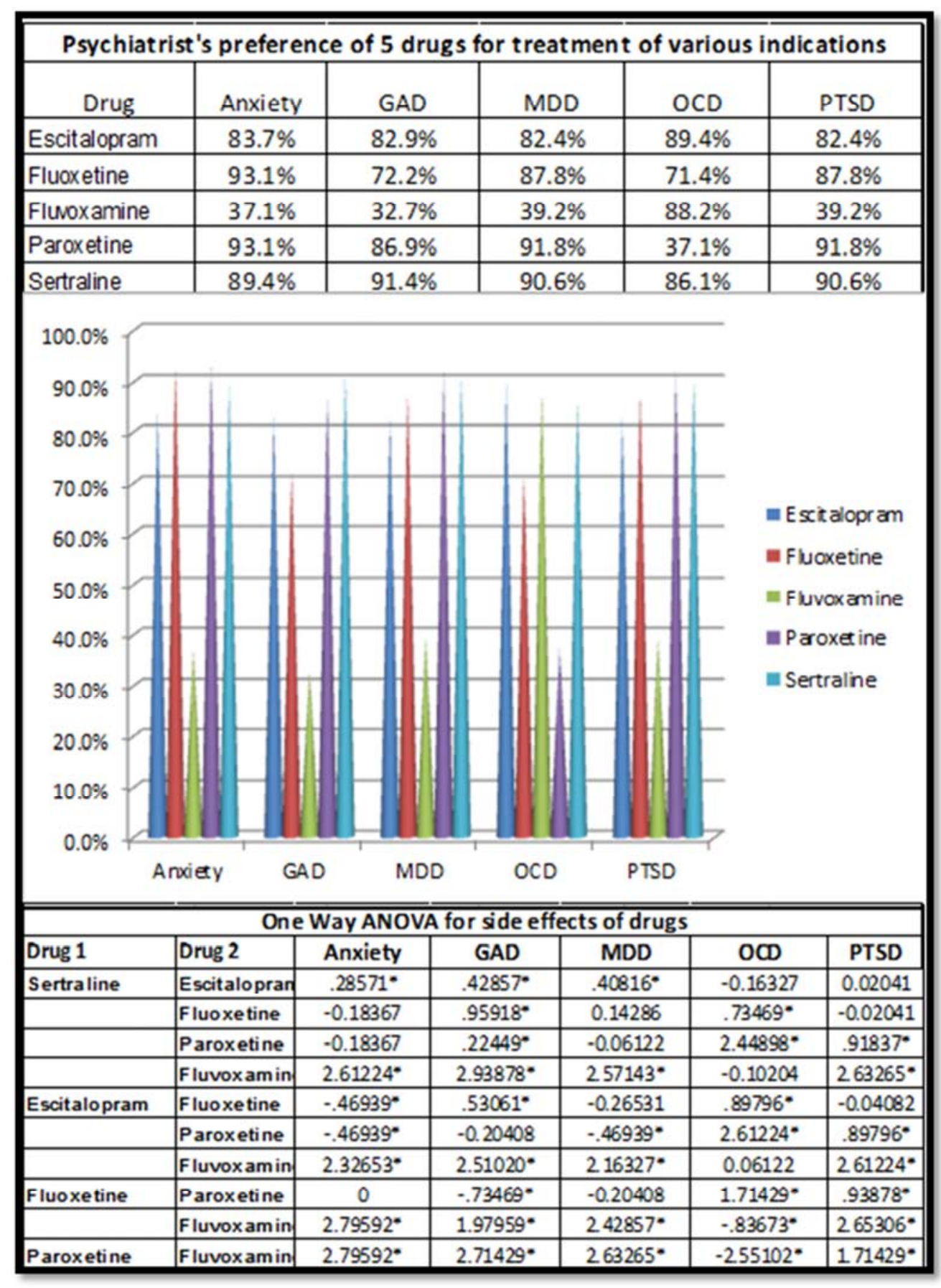

Figure 2. Preference scores and bar charts for prescribed uses of the SSRIs under study.

\subsection{Side Effects}

Refer to figure 3. The experience/opinion of psychiatrists on each type of side effect under study arising from use of the 5 drugs was significant. Figure shows the \% score given by psychiatrists to drugs under study, the clustered bar charts and one way ANOVA for the various side effects under consideration. Except for sexual dysfunction, significant differences were observed in experience/opinion about occurrence of side effects. 


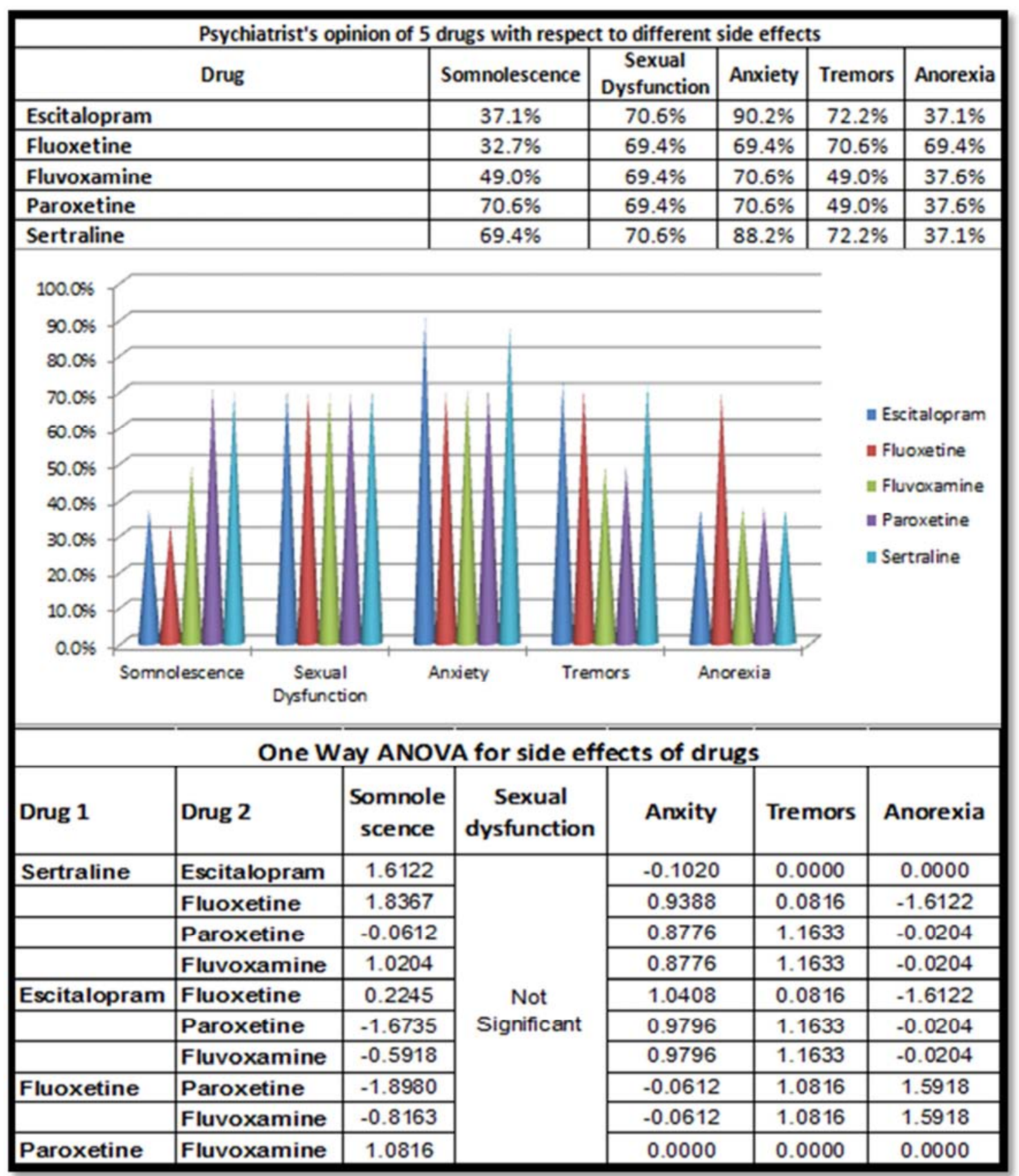

Figure 3. Scores given by Psychiatrists, bar charts and one way anova for the observed side effects of the SSRIs under study.

\subsection{Patient Demographics}

\subsubsection{Patient's Age}

Refer to figure 4. Significant preferences for all drug molecules for the classes were observed. Figure shows the \% preference score, the clustered bar charts and one way ANOVA for the two age classes under consideration. Significant mean differences were observed in preferences for the 5 drugs in both the age classes when variances within the group were tested using one-way ANOVA. 


\begin{tabular}{|c|c|c|c|}
\hline \multicolumn{4}{|c|}{ Psychiatrist's preferences with respect to special Age Class } \\
\hline Drug & \multicolumn{2}{|c|}{ Geriatric Patient } & Adolescent Patient \\
\hline Escitalopram & \multicolumn{2}{|c|}{$88.6 \%$} & $89.4 \%$ \\
\hline Fluoxetine & \multicolumn{2}{|c|}{$69.8 \%$} & $74.3 \%$ \\
\hline Fluvoxamine & \multicolumn{2}{|c|}{$80.0 \%$} & $83.3 \%$ \\
\hline Paroxetine & \multicolumn{2}{|c|}{$68.6 \%$} & $93.9 \%$ \\
\hline Sertraline & \multicolumn{2}{|c|}{$91.0 \%$} & $86.5 \%$ \\
\hline \multicolumn{4}{|c|}{ 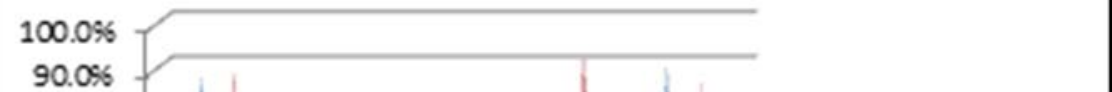 } \\
\hline \multicolumn{4}{|c|}{$80.0 \% 6+||$} \\
\hline \\
\hline \multirow{5}{*}{\multicolumn{4}{|c|}{$\begin{array}{l}\text { = Geriatric Patient } \\
\text { = Adolscent Patient }\end{array}$}} \\
\hline & & & \\
\hline & & & \multirow[t]{2}{*}{ Idolscent Patient } \\
\hline & & & \\
\hline & & & \\
\hline \multicolumn{4}{|c|}{ One Way ANOVA for Age Groups } \\
\hline Drug 1 & Drug 2 & $\begin{array}{l}\text { Geriatric } \\
\text { Patlent }\end{array}$ & $\begin{array}{l}\text { Adolscent } \\
\text { Patient }\end{array}$ \\
\hline \multirow[t]{4}{*}{ Sertraline } & Escitalopram & 0.12245 & -0.14286 \\
\hline & Fluoxetine & $1.06122^{*}$ & $.61224^{*}$ \\
\hline & Paroxetine & $1.12245^{*}$ & $-.36735^{*}$ \\
\hline & Fluvoxamine & $.55102^{*}$ & 0.16327 \\
\hline \multirow[t]{3}{*}{ Escitalopram } & Fluoxetine & $.93878^{*}$ & $.75510^{*}$ \\
\hline & Paroxetine & $1.00000^{*}$ & -0.22449 \\
\hline & Fluvoxamine & $42857^{*}$ & $.30612^{*}$ \\
\hline Fluoxetine & Paroxetine & 0.06122 & $-.97959^{*}$ \\
\hline & Fluvoxamine & $-.51020^{*}$ & $-.44898^{*}$ \\
\hline Paroxetine & Fluvoxamine & $-.57143^{*}$ & $53061^{*}$ \\
\hline
\end{tabular}

Figure 4. Preference scores of Psychiatrists for drugs under study, clustered bar charts and one way ANOVA for the age groups under study.

\subsubsection{Gender of Patients}

Refer to figure 5. Preferences for all drug molecules were significant for both male and female patients. Figure 6 shows the psychiatrist's preference scores (\%), the clustered bar charts and one way ANOVA of the drugs under study for male and female patients respectively. One way ANOVA for female patients turned out to be non-significant whereas for male patients it was found to be significant. 


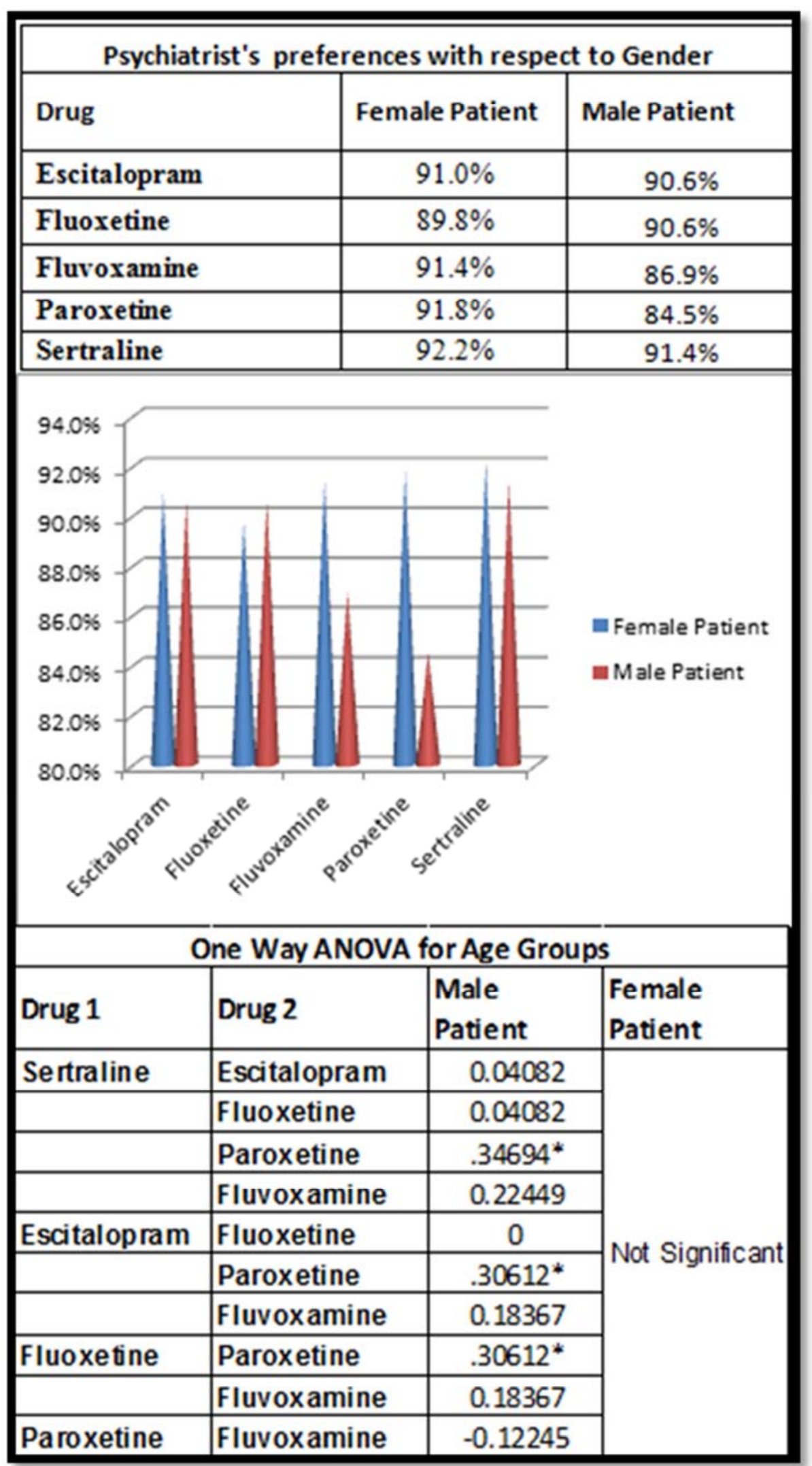

Figure 5. Preference scores, clustered bar charts and one-way ANOVA for the preferred SSRI with respect to gender.

\subsection{Commercial Factors}

The overall ranking of the 4 marketing related factors with respect to the importance given by psychiatrists while prescribing 
SSRI was derived from the individual psychiatrists' ranking responses. The factors considered were availability of the drug, cost of the drug, and brand value of the drug and the interpersonal skills of the medical representative. Refer Figure 6 for the bar chart.

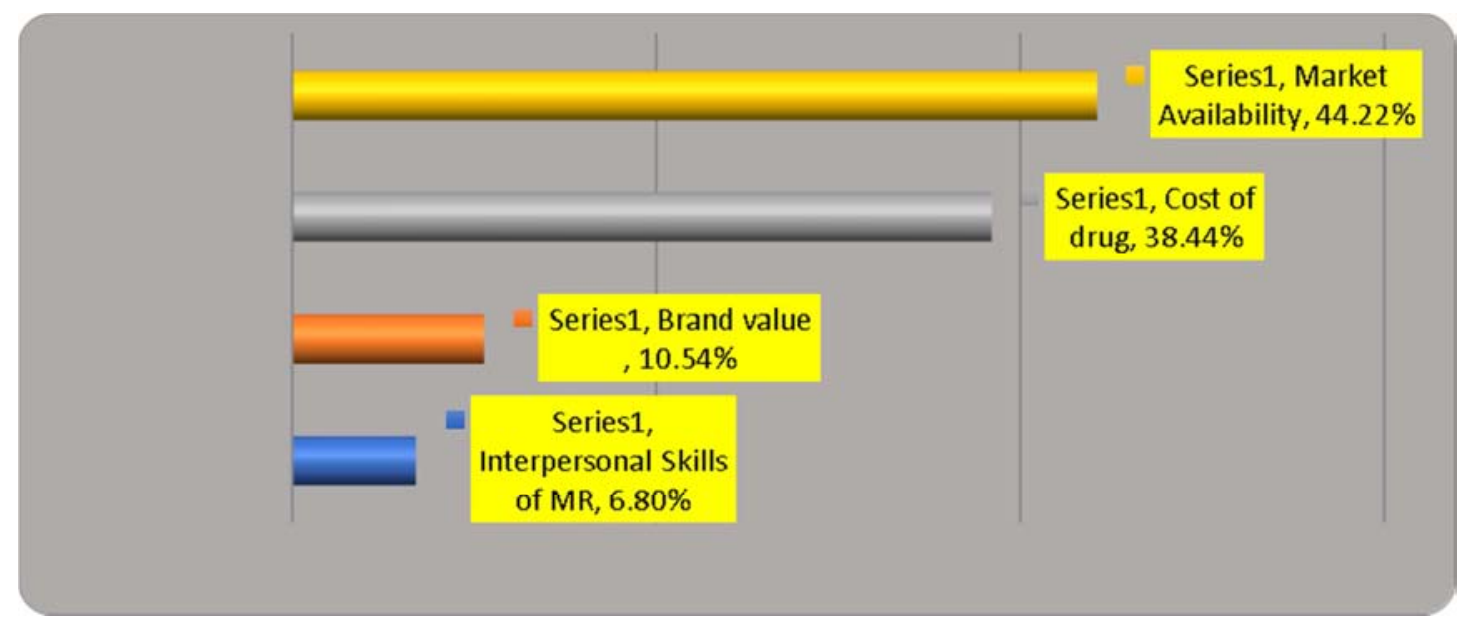

Figure 6. Bar chart of the markting factors with respect to the importance given by psychiatrists while prescribing SSRI.

\section{Discussion}

\subsection{Pharmacokinetics}

Psychiatrists experience/opinion wrt the contra indications, efficacy, onset of action, duration of action and bioavailability of the 5 drug molecule brands under study are significant.

Refer to figure 1, for performance of the drugs w.r.t. contra indications. Use of Escitalopram witnesses least contra indications whereas use of Fluvoxamine risks maximum contra indications.

Considering duration of action of the drugs, Sertraline has the highest duration of action whereas Fluoxetine, Paroxetine and Fluvoxamine display least duration of action.

With respect to efficacy, bioavailability and onset of action, there are no significant differences within the 5 drugs under study.

\subsection{Uses}

Refer to figure 2. For the treatment of seasonal affective disorder (SAD) Fluoxetine along with Paroxetine are the most preferred whereas Fluvoxamine is the least preferred drug.

For the treatment of generalized anxiety disorder (GAD) Sertraline is the most preferred whereas Fluvoxamine is the least preferred drug molecule brand.

For the treatment of major depressive disorder (MDD) Paroxetine is the most preferred whereas Fluvoxamine is the least preferred drug molecule brand.

For the treatment of obsessive compulsive disorder (OCD) Escitalopram is the most preferred whereas Paroxetine is the least preferred drug molecule brand.

For the treatment of Post-Traumatic Stress disorder (PTSD) Fluoxetine along with Sertraline is the most preferred whereas Fluvoxamine is the least preferred drug molecule brand.

\subsection{Side Effects}

Refer to figure 3. Paroxetine has the highest risk of patient experiencing the side effect of somnolence where as it is least for Fluoxetine.

Escitalopram has the highest risk of patient experiencing the side effect of anxiety where as it is least for Fluoxetine.

Sertraline and Escitalopram have the highest risk of patient experiencing the side effect of tremors where as it is least for Paroxetine and Fluvoxamine.

Fluoxetine has the highest risk of patient experiencing the side effect of anorexia where as it is least for drugs Sertraline and Escitalopram.

With reference to the side effect of sexual dysfunction, there are no significant differences amongst the drugs.

\subsection{Patient Demographics}

\subsubsection{Patient's Age}

For treatment of geriatric patients all the 5 antidepressant drug molecules under study were used by psychiatrists. Refer to figure 5 for $\%$ preference scores. Sertraline was the most preferred, whereas paroxetine was the least preferred drug. Also, psychiatrists indicated moderate but considerable preference for the drug fluvoxamine. Psychiatrists displayed high preference with negligible difference between drugs sertraline and escitalopram, although they displayed low preference with negligible difference between drugs fluoxetine and paroxetine.

For treatment of adolescent patients all the 5 antidepressant drug molecule drugs under study were used by psychiatrists. Refer to figure 4 for the \% preference scores. Paroxetine was the most preferred drug whereas fluoxetine was the least preferred drug. Drug pairs with insignificant difference in preference of psychiatrists were: sertraline and escitalopram, sertraline and fluvoxamine and escitalopram and paroxetine. 


\subsubsection{Patient's Gender}

Psychiatrists used all 5 drug molecules under study for treatment of male patients. Refer to figure 5. Sertraline, Escitalopram and Fluoxetine were equally preferred. Paroxetine was the least preferred but was not far behind on the preference scores. Drug pairs with significant differences were: Paroxetine with each one of Sertraline, Escitalopram and Fluoxetine.

Psychiatrists used all 5 drug molecules under study for the treatment of female patients. Refer to figure 5 . All the 5 drug molecules were almost frequently preferred with no significant differences amongst them.

\subsection{Ranking of Commercial Factors}

Referring to figure 6 , amongst the four commercial factors, ease of availability of drug in the market was considered as the most important factor, followed closely by the cost of drug. Brand value and interpersonal skills of the MR were rated to be relatively of low importance.

\section{Difference Between Literature and Psychiatrist Perceptions/ Practice}

\subsection{Pharmacokinetics}

\subsubsection{Contra-Indications}

Theoretically, all SSRIs have the same number of contraindications, since they have the same mechanism of action, though fluoxetine and paroxetine have a relatively higher potential of drug-drug interactions. Nonetheless, escitalopram was perceived to have the lowest, while fluvoxamine was perceived to have the highest number of contra-indications.

\subsubsection{Duration of Action}

There is no set difference between the duration of action of the 5 drugs under study, although, according to secondary research, $1 / 3^{\text {rd }}$ of the total therapeutic effect at six weeks was apparent by the $1^{\text {st }}$ week of treatment. Also, fluoxetine has the highest half-life at 2-4 days, while that of paroxetine is the least, at 16 hours. Yet, based on the study, Sertraline was perceived to have the highest duration of action.

\subsection{Uses}

There were many deviations from literature found with respect to the uses of the drugs. Refer to figure 7 . The column of 'literature drugs' enlists the drugs to be used for a particular type of disorder, while that of 'perceived drugs' enlists the drugs perceived to be used for the particular disorder type.

\subsection{Side Effects}

There were a few deviations from literature found with respect to the side effects of the drugs. Refer to figure 7 . The column of 'literature drugs' enlists the drugs that are known to cause the side effect under consideration, while that of 'perceived drugs' enlists the drugs perceived to cause the particular side effect.

\begin{tabular}{|l|l|l|l|}
\hline & & Literature Drugs & Perceived Drugs \\
\hline \multirow{4}{*}{$\begin{array}{l}\text { Disorder } \\
\text { Type }\end{array}$} & SAD & Sertraline, fluvoxamine & Fluoxetine, paroxetine \\
\cline { 2 - 4 } & GAD & Escitalopram & Sertraline \\
\cline { 2 - 4 } & GDD & All five drugs under study & Paroxetine \\
\cline { 2 - 4 } & OCD & Fluoxetine, paroxetine, sertraline & Escitalopram \\
\cline { 2 - 4 } & PTSD & Fluoxetine, paroxetine, sertraline & Fluoxetine, sertraline \\
\hline \multirow{3}{*}{$\begin{array}{l}\text { Side } \\
\text { Effect }\end{array}$} & Somnolence & Fluvoxamine & Paroxetine \\
\cline { 2 - 4 } & Anxiety & Fluoxetine, Fluvoxamine & Escitalopram \\
\cline { 2 - 4 } & Tremors & Fluvoxamine & Sertraline, escitalopram \\
\hline
\end{tabular}

Figure 7. Table giving the difference between literature and perception / practice of psychiatrists with respect to uses and side effects of SSRIS.

\subsection{Patient demographics}

\subsubsection{Preference of Drugs for Geriatric \& Adolescent Class Patients}

1. Geriatrics: Based on the secondary research undertaken, no bias was found for the prescription of drugs to geriatrics patients. During this study it was found that sertraline was the most preferred drug for prescription to geriatric patients, while paroxetine was the least preferred.

2. Adolescent: No literature was found to support a bias for prescription of SSRIs to adolescents, yet, through this study, paroxetine was found to be the most preferred, while fluoxetine was considered to be the least preferred drug.

\subsubsection{Preference with Respect to Gender of Patients}

1. Males: No literature was found showing a bias of SSRI prescription to males, yet sertraline, fluoxetine and escitalopram were preferred almost equally, much higher than the other drugs in the study.

2. No such bias was observed for prescription to females.

\section{Managerial Implications and Recommendations}

This paper enlists the differences observed in the literature and actual preferences of psychiatrists with respect to:

1. Presence of patient in special age groups (adolescent/ 
geriatric).

2. Gender of the patient.

3. Uses of SSRI for a particular indication.

It also helps ranking the commercial aspects of SSRIs.

For example, it is perceived that use of Escitalopram has the highest chance of showing anxiety as a side effect, which is not the case based on the literature. Due to this incorrect perception, a large market of patients suffering from both anxiety and depression are not reached. Firms could thus realign their strategies to better suit the market needs.

\section{Conclusion}

There exists a gap between the theoretical evidence and the practical observations about the pharmacokinetics, uses and side effects of the 5 SSRIs under study. For example, sertraline, a drug proven to be useful in the treatment of depression, has an incorrect bias of causing anxiety as a side effect. This results in psychiatrists not prescribing an effective drug due to a bias with no theoretical basis, thus resulting in poorer treatment outcomes for the patient. For healthcare organizations, it means that they are losing out on almost half the potential patient population. Therefore, it would be useful for them to undertake communication campaigns that revolved around thwarting such biases, thus resulting in better treatment choices for patients and improved health outcomes.

\section{Limitations of the Study}

The study was conducted with the help of 49 psychiatrists, of 186 psychiatrists practicing in Mumbai region. Due to constraint of resources psychiatrists practicing in other metros and Tier $2 \& 3$ cities could not be included in the study. Psychiatrist perceptions and practice may differ significantly based on the location of their practice.

\section{References}

[1] (n. d.). Retrieved Novemeber $19^{\text {th }}$, 2018, from Depression statistics WHO India: http://www.searo.who.int/india/topics/depression/en/.

[2] Nutt, D. J. (2005, Jan). Overview of Diagnosis and Drug Treatments of Anxiety Disorders. CNS Spectums, 10 (1), 4956.
[3] Sheehan, D. V. (2006). Assessing Remission In Major Depressive Disorder And Generalized Anxiety Disorder Clinical Trials With The Discan Metric Of The Sheehan Disability Scale. . International Clinical Pharmacology.

[4] Renoir, T. (2013). Selective Serotonin Reuptake Inhibitor Antidepressant Treatment Discontinuation Syndrome: A Review Of The Clinical Evidence And The Possible Mechanisms Involved. Frontiers In Pharmacology.

[5] Tyler, M. F. (2006). Early Onset Of SSRI Antidepressant Action. . Europe PMC Funders Group.

[6] Harten, J. V. (1993, March). Clinical Pharmacokinetics Of SSRIs. Clinical Pharmacokinetics, 24 (3), 203-220.

[7] Andre Tylee, P. W. (2007). Onset of action of antidepressants. British Medicine Journal, 334-911.

[8] Ferguson, J. (2001, February). SSRI Antidepressant Medications: Adverse Effects And Tolerability. The Primary Care Companion To The Journal Of Clinical Psychiatry., 3 (1), 22-27.

[9] Trivedi, J. K. (2010). Antidepressant Drug Prescription Patterns For Depression At A Tertiary Health Care Centre Of Northern India. Medical Practice And Review.

[10] Marco Piccinelli, G. W. (2000, December). Gender differences in depression: Critical review. The British Journal of Psychiatry, 177 (6).

[11] Marken P, M. (2000, ecember). Selecting A Selective Serotonin Reuptake Inhibitor: Clinically Important Distinguishing Factors. The Journal Of Clinical Psychiatry, 2 (6), 205-210.

[12] Cohen, D. (2007). Should The Use Of SSRIs In Child And Adolescent Depression Be Banned? . Psychotherapy And Psychosomatics., 76 (1), 5-14.

[13] Sanchez, C. R. (2014, July). A Comparative Review Of Escitalopram, Paroxetine, And Sertraline: Are They All Alike? . International Clinical Psychopharmacology., 24 (5), 185-196.

[14] Huskamp, H. D. (2003, August). Pharmacoeconomics. . 26 (7), 603-616.

[15] List of Essential Medicines from WHO. (2015). Retrieved from http://apps.who.int/medicinedocs/en/m/abstract/Js23088en/. 